\title{
APPLICATION OF FRACTIONAL ORDER THEORY OF THERMOELASTICITY IN AN ELLIPTICAL DISK AND ASSOCIATED THERMAL STRESSES
}

\author{
S. THAKARE ${ }^{*}$ and Y. PANKE \\ Department of Mathematics, K.I.T.S. \\ Ramtek, INDIA \\ E-mail: shivthakre5@gmail.com \\ K. HADKE \\ Department of Mathematics \\ R.G.C. Engineering \& Research \\ Nagpur, INDIA
}

\begin{abstract}
In this article, a time fractional-order theory of thermoelasticity is applied to an isotropic homogeneous elliptical disk. The lower and upper surfaces of the disk are maintained at zero temperature, whereas the sectional heat supply is applied on the outer curved surface. Thermal deflection and associated thermal stresses are obtained in terms of Mathieu function of the first kind of order $2 n$. Numerical evaluation is carried out for the temperature distribution, Thermal deflection and thermal stresses and results of the resulting quantities are depicted graphically.
\end{abstract}

Key words: elliptical disk, fractional-order theory, Caputo derivative, integral transform, thermal stresses, thermal deflection.

\section{Introduction}

Fractional calculus is a very useful and interesting subject for mathematicians. It is a generalization of the concept of ordinary differentiation $D^{\alpha} f(t)$ to non integer values of $\alpha$ that goes back to the beginning of the concept of differential calculus. Many models of physical processes such as heat conduction, wave propagation, diffusion, electric theories and viscoelasticity have been modified by fractional calculus. In the second half of the 19th century the theory of fractional integrals and derivatives was established. Abel [1] was first to apply fractional derivatives during the formulation of the Tautochrone problem by using fractional calculus in the solution of an integral equation. Caputo and Mainardi $[2,3]$ and Caputo [4] introduced a model of dissipation based on memory. Ning [5] used the variable separation method to obtain an analytical solution for the time-fractional heat conduction equation in the spherical coordinate system.

Lenzi et al. [6] studied exact solutions of the fractional diffusion equation by Green function approach. Povstenko [7,8] investigated variation of time-fractional differential operators with memory effects. Also, Povstenko [9] solved analytically fractional heat conduction in a space with a source varying harmonically in time and calculated associated thermal stresses. Abbas [10] studied the fractional order theory with thermoelastic diffusion using the Laplace and Eigen value approach for an infinite elastic medium within a spherical cavity.

Deshmukh [11] solved a two dimensional thermoelastic problem of a thin circular plate by a quasistatic approach in the context of the fractional order theory. Youssef [12] in 2010 introduced the generalized

\footnotetext{
* To whom correspondence should be addressed
} 
thermoelastic theory for heat conduction in the context of the fractional integral. Also, the uniqueness of the solutions is proved in [13]. Lamba [14] presented mathematical modeling of thermoelastic hollow cylinder subjected to convective boundary condition on the upper and lower plane surface by the application of the fractional order theory. Tripathi [15] studied a dynamic thermoelastic problem of fractional order for a thick circular plate with finite wave speeds. Kukla [16] presented an analytical solution to the problem of timefractional heat conduction in a sphere. Lamba [17] applied the fractional order theory to determine thermal deflection and stresses of a circular disk subjected to axisymmetric and partially distributed heat supply on the upper surface while the lower surface is kept thermally insulated. Lamba [18] applied the fractional order theory to analyze the temperature distribution, displacement, thermal stresses function and deflection on the outer curved surface of a solid circular cylinder.

Due to the advantage of physical, mechanical, as well as thermal properties of elliptical structures materials, a copper (pure) elliptical disk is used extensively and much more frequently in modern engineering applications as compared to other shape objects. Both analytical and numerical techniques are the best methodology to solve such problems. But there are only few studies concerned with elliptical objects for both steady and transient state heat conduction problems. McLachlan $[19,20]$ solved the heat conduction problem for an elliptical cylinder in the form of an infinite Mathieu function series. Dhakate [21] determined thermally induced transverse vibration of a uniform thin elliptical disk with elastic supports at both radial boundaries by using the integral transform technique. Gupta [22] determined the boundary value problem for elliptic cylinders by introducing a finite transform involving Mathieu functions. Bhad [23] investigated the thermoelastic problems on an elliptical plate in which interior heat sources are generated within the solid. Dhakate [24] used Berger's approximate methods to determine the large deflection of a heated thin annular sector plate with clamped edges under transient temperature distribution.

From the above a literature it is clear that till now no work has dealt with the thermoelastic bending analysis in the context of the fractional-order theory of thermoelasticity. So the authors have been motivated to conduct this study. In this paper, a mathematical model of a fractional-order thermoelastic problem for a thin simply supported elliptical disk subjected to sectional heat supply on the outer curved surface is developed. The numerical computations have been carried out for a copper (pure) elliptical disk and the results for temperature distribution, thermal deflection, and stresses are discussed and illustrated graphically.

\section{Formulation of the problem}

By following Varghese [21], here we consider a thin elliptical disk (as shown in Fig.1) with the space variables $(\xi, \eta, z)$ of thickness $\ell$ occupying space $D$ defined by $\xi_{i}<\xi<\xi_{o}$ and $0<\eta<2 \pi$, whose lower and upper surfaces are maintained at zero temperature, whereas the sectional heat supply is applied on the outer curved surface.

The relation between Cartesian and elliptical coordinates is defined as

$$
x=c \cosh \xi \cos \eta, \quad y=c \sinh \xi \sin \eta, \quad z=z, h^{2}=2 /\left[c^{2}(\cosh 2 \xi-\cos 2 \eta)\right] .
$$

By using the transformation $\xi+i \eta=\cosh ^{-1}[(x+i y) / c]$ where $2 c=2 \sqrt{a_{o}^{2}-b_{o}^{2}}=2 \sqrt{a_{i}^{2}-b_{i}^{2}}$ denotes the distance between their common foci. The semi-major axis varies from the inner diameter $2 a_{i}$ to the outer diameter $2 a_{o}$ and the semi-minor axis varies from the inner diameter $2 b_{i}$ to the outer diameter $2 b_{o}$. Whereas $\xi \in\left(\xi_{i}, \xi_{o}\right)$ and at the inner and outer elliptical boundary are given as $\xi_{i}=\tanh ^{-1}\left(b_{i} / a_{i}\right)$ $\xi_{o}=\tanh ^{-1}\left(b_{o} / a_{o}\right)$ respectively.

A mathematical model is prepared considering a nonlocal Caputo type time fractional heat conduction equation of order $\alpha$ for a thin elliptical disk.

The definition of the Caputo type fractional derivative is given by [25] 


$$
\frac{d^{\alpha} f(t)}{d t^{\alpha}}= \begin{cases}\frac{1}{\Gamma(n-\alpha)} \int_{0}^{t}(t-\tau)^{n-\alpha-1} \frac{d^{n} f(\tau)}{d \tau^{n}} d \tau, & n-1<\alpha<n, \\ \frac{d^{n} f(\tau)}{d t^{n}}, & \alpha=n\end{cases}
$$

with the following Laplace transform rule

$$
L\left\{\frac{d^{\alpha} f(t)}{d t^{\alpha}}\right\}=s^{\alpha} L\{\bar{f}(s)\}-\sum_{k=0}^{n-1} f^{(k)}\left(0^{+}\right) s^{\alpha-1-k}, \quad n-1<\alpha<n
$$

in which $s$ is the transform parameter.

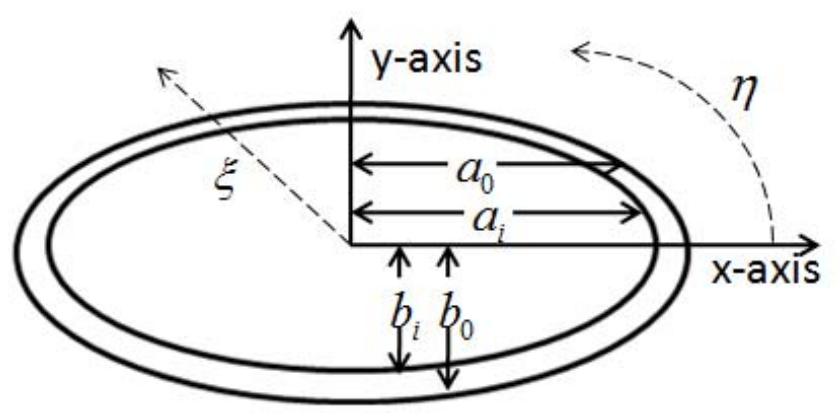

Fig.1. Geometry of a thin elliptical disk.

\section{Temperature distribution}

The temperature of the disk $\theta(\xi, \eta, z, t)$ satisfies the time fractional order differential equation (Following [8] and [21])

$$
\left\{h^{2}\left(\frac{\partial^{2}}{\partial \xi^{2}}+\frac{\partial^{2}}{\partial \eta^{2}}\right)+\frac{\partial^{2}}{\partial z^{2}}\right\} \theta(\xi, \eta, z, t)=\frac{1}{\kappa} \frac{\partial^{\alpha}}{\partial t^{\alpha}} \theta(\xi, \eta, z, t)
$$

subjected to boundary and initial conditions

$$
\left.\begin{array}{l}
\left.\theta(\xi, \eta, z, t)\right|_{t=0}=0 \quad ; \quad 0<\alpha \leq 2 \\
\left.\frac{\partial \theta}{\partial t}(\xi, \eta, z, t)\right|_{t=0}=0 ; \quad 1<\alpha \leq 2 \\
\left.\theta(\xi, \eta, z, t)\right|_{\xi=\xi_{i}}=0,\left.k D_{R L}^{I-\alpha} \frac{\partial \theta}{\partial \xi}(\xi, \eta, z, t)\right|_{\xi=\xi_{0}}=f_{l}(z) \delta(t) \\
\left.\theta(\xi, \eta, z, t)\right|_{z=0}=0,\left.\quad \theta(\xi, \eta, z, t)\right|_{z=\ell}=0
\end{array}\right\}
$$


where $\kappa=\lambda / \rho C$ represents thermal diffusivity, $\lambda$ is the thermal conductivity of the material, $\rho$ is the density and $C$ is the calorific capacity.

\section{Thermal deflection and thermal stresses}

The expressions of thermally induced resultant force and resultant moment are given as [21]

$$
M_{\theta}(\xi, \eta, t)=\alpha E \int_{0}^{\ell} z \theta(\xi, \eta, z, t) d z, \quad N_{\theta}(\xi, \eta, t)=\alpha E \int_{0}^{\ell} \theta(\xi, \eta, z, t) d z
$$

where $E$ denotes the Young modulus and $\alpha$ is the coefficient of linear thermal expansion of the material of the disk, respectively.

The governing differential equation for the disk in terms of normal deflection $\omega(\xi, \eta, t)$ is

$$
\left(\frac{\partial^{2}}{\partial \xi^{2}}+\frac{\partial^{2}}{\partial \eta^{2}}\right)^{2} \omega(\xi, \eta, t)=-\frac{1}{2 b^{2} h^{4} \rho \ell(1-v)} \nabla^{2} M_{\theta}(\xi, \eta, t)
$$

where

$$
b^{2}=D / 2 \rho \ell
$$

here $D$ denotes the flexural stiffness of the disk and is defined as $D=E \ell^{3} / 12\left(1-v^{2}\right)$.

The basic relations of resultant bending moments per unit width $\left(M_{i j}\right)$, resultant forces $\left(N_{i j}, i, j=\xi, \eta\right)$ are defined [23] as

$$
\begin{aligned}
& N_{\xi}=N_{\eta}=N_{\xi \eta}=0, \\
& M_{\xi}=-h^{2} D\left\{\left(\frac{\partial^{2} \omega}{\partial \xi^{2}}+v \frac{\partial^{2} \omega}{\partial \eta^{2}}\right)-\frac{c^{2} h^{2}(1-v)}{2}\left[\sinh 2 \xi \frac{\partial \omega}{\partial \xi}-\sin 2 \eta\right] \frac{\partial \omega}{\partial \eta}\right\}-\frac{M_{\theta}}{1-v}, \\
& M_{\eta}=-h^{2} D\left\{\left(v \frac{\partial^{2} \omega}{\partial \xi^{2}}+\frac{\partial^{2} \omega}{\partial \eta^{2}}\right)+\frac{c^{2} h^{2}(1-v)}{2}\left[\sinh 2 \xi \frac{\partial \omega}{\partial \xi}-\sin 2 \eta\right] \frac{\partial \omega}{\partial \eta}\right\}-\frac{M_{\theta}}{1-v}, \\
& M_{\xi \eta}=-h^{2} D(1-v)\left\{\frac{\partial \omega}{\partial \xi} \sin 2 \eta+\frac{\partial \omega}{\partial \eta} \sinh 2 \xi-\frac{2}{c^{2} h^{2}} \frac{\partial^{2} \omega}{\partial \xi \partial \eta}\right\} .
\end{aligned}
$$

The aforementioned Eq.(4.2) and first equation of (4.4) must satisfy the initial condition

$$
\begin{aligned}
& \left.\omega\right|_{t=0}=\left.\frac{\partial \omega}{\partial t}\right|_{t=0}=0 \quad \text { for } \quad \xi_{i} \leq \xi \leq \xi_{o}, \quad 0 \leq \eta \leq 2 \pi, \\
& \left.\omega\right|_{\xi=\xi_{i}}=\left.M_{\xi}\right|_{\xi=\xi_{i}}=0 \quad \text { for all } \quad \eta \quad \text { in } \quad 0 \leq \eta \leq 2 \pi .
\end{aligned}
$$


The stress functions $\sigma_{\xi \xi}, \sigma_{\eta \eta}$ and $\sigma_{\xi \eta}$ in terms of resultant forces and resultant moments are given $[23-24]$ as

$$
\begin{aligned}
& \sigma_{\xi \xi}=\frac{1}{\ell} N_{\xi}+\frac{12 z}{\ell^{3}} M_{\xi}+\frac{1}{1-v}\left(\frac{1}{\ell} N_{\theta}+\frac{12 z}{\ell^{3}} M_{\theta}-\alpha E \theta\right), \\
& \sigma_{\eta \eta}=\frac{1}{\ell} N_{\eta}+\frac{12 z}{\ell^{3}} M_{\eta \eta}+\frac{1}{1-v}\left(\frac{1}{\ell} N_{\theta}+\frac{12 z}{\ell^{3}} M_{\theta}-\alpha E \theta\right), \\
& \sigma_{\xi \eta}=\frac{1}{\ell} N_{\xi \eta}-\frac{12 z}{\ell^{3}} M_{\xi \eta} .
\end{aligned}
$$

Equations (2.1) to (4.7) constitute the mathematical formulation of the problem under consideration.

\section{Solution to the problem}

\subsection{Solution for temperature distribution}

To obtain the expression for the temperature function $\theta(\xi, \eta, z, t)$, we use the finite Fourier cosine transformation with respect to the variable $z$ and then introduce the finite integral transform ([22] and [21]) involving Mathieu functions as

$$
\bar{f}\left(q_{2 n, m}\right)=\int_{\xi_{i}}^{\xi_{o}} \int_{0}^{2 \pi} f(\xi, \eta)(\cosh 2 \xi-\cos 2 \eta) B e_{2 n}\left(\xi, q_{2 n, m}\right) c e_{2 n}\left(\eta, q_{2 n, m}\right) d \xi d \eta
$$

with its inversion theorem at any point within the range as

$$
\begin{aligned}
& f(\xi, \eta)=\sum_{n=0}^{\infty} \sum_{m=1}^{\infty} \frac{\bar{f}\left(q_{2 n, m}\right) B e_{2 n}\left(\xi, q_{2 n, m}\right) c e_{2 n}\left(\eta, q_{2 n, m}\right)}{\pi \int_{\xi_{i}}^{\xi_{o}}\left(\cosh 2 \xi-\Theta_{2 n, m}\right) B e_{2 n}^{2}\left(\xi, q_{2 n, m}\right) d \xi} \\
& \Theta_{2 n, m}=\frac{1}{\pi} \int_{0}^{2 \pi} \cos 2 \eta c e_{2 n}^{2}\left(\eta, q_{2 n, m}\right) d \eta=A_{0}^{(2 n)} A_{2}^{(2 n)}+\sum_{r=0}^{\infty} A_{2 r}^{(2 n)} A_{2 r+2}^{(2 n)}
\end{aligned}
$$

in these series $A$ represents the functions of $q, q_{2 n, m}$ is a root of the transcendental equation $B e_{2 n}\left(\xi_{0}, q_{2 n, m}\right)=0$, and having the kernel as

$$
B e_{2 n}\left(\xi, q_{2 n, m}\right)=C e_{2 n}\left(\xi, q_{2 n, m}\right) F e y_{2 n}^{\prime}\left(\xi_{i}, q_{2 n, m}\right)-F e y_{2 n}\left(\xi, q_{2 n, m}\right) C e_{2 n}^{\prime}\left(\xi_{i}, q_{2 n, m}\right),
$$

in which prime dash denotes differentiation with respect to the variable $\xi, c e_{2 n}(\eta, q)$ is the ordinary Mathieu function [20] of first kind of order $n, C e_{2 n}(\xi, q)$ is a modified Mathieu function [20] of first kind of order $n$ and the recurrence relations for the Bessel functions $Y_{2 r}$ (are identical in form with those for $J_{2 r}$ ) can be defined [20] as 


$$
F e y_{2 n}(\xi, q)=\frac{c e_{2 n}(0, q)}{A_{0}^{(2 n)}} \sum_{r=0}^{\infty} A_{2 r}^{(2 n)} Y_{2 r}\left(2 k^{\prime} \sinh \xi\right)\left(\begin{array}{c}
|\sinh \xi|>1 \\
R(\xi)>0
\end{array}\right)
$$

with $y$ in Fey which signifies the $Y$ - Bessel function and $q=k^{2}=\lambda c^{2} / 4$ [20].

Applying the finite integral transform [22] given in Eq.(5.1) and finite Fourier cosine transformation and their inversions to Eq.(2.3) and making use of the transformed boundary and initial conditions (2.4), one obtains the temperature distribution function expressed as

$$
\begin{aligned}
& \theta(\xi, \eta, z, t)=\frac{2}{\ell} \sum_{n=0}^{\infty} \sum_{m=1}^{\infty} \sum_{p=1}^{\infty} \cos \left(\beta_{p} z\right)\left\langle\int_{0}^{t} E_{\alpha}\left\{\left[-\kappa\left(\lambda_{2 n, m}^{2}+\beta_{p}^{2}\right)\right] u^{\alpha}\right\}\right. \\
& \left.\times\left[-\frac{4 \kappa}{c^{2}} \pi A_{0}^{2 n} B e_{2 n}\left(\xi_{0}\right) \hat{\bar{f}}\left(q_{2 n, m}, \beta_{p}, t-u\right)\right] d u\right\rangle \times B e_{2 n}\left(\xi, q_{2 n, m}\right) c e_{2 n}\left(\eta, q_{2 n, m}\right) / C_{2 n, m}
\end{aligned}
$$

where

$$
E_{\alpha}\left[-\kappa\left(\lambda_{2 n, m}^{2}+\beta_{p}{ }^{2}\right)\right] t^{\alpha}=L^{-1}\left\{\frac{1}{s^{\alpha}+\kappa\left(\lambda_{2 n, m}^{2}+\beta_{p}{ }^{2}\right)}\right\} .
$$

Here, $E_{\alpha}(z)$ represents the Mittag-Leffler-type function [25] described by the series representation

$$
E_{\alpha}(z)=\sum_{n=0}^{\infty} \frac{z^{n}}{\Gamma(\alpha n+1)}, z \in C, \operatorname{Re}(\alpha)>0
$$

\subsection{Solution for thermal deflection and stresses}

Using Eqs (5.3) in Eq.(4.1), we get the resultant moment and resultant force as follows

$$
\begin{aligned}
& M_{\theta}=\frac{2 \alpha E}{\ell} \sum_{n=0}^{\infty} \sum_{m=1}^{\infty} \sum_{p=1}^{\infty}\left[(-1)^{p}-1\right]\left\langle\int_{0}^{t} E_{\alpha}\left\{\left[-\kappa\left(\lambda_{2 n, m}^{2}+\beta_{p}^{2}\right)\right] u^{\alpha}\right\} \times\right. \\
& \left.\times\left[-\frac{4 \kappa}{c^{2}} \pi A_{0}^{2 n} B e_{2 n}\left(\xi_{0}\right) \hat{\bar{f}}\left(q_{2 n, m}, \beta_{p}, t-u\right)\right] d u\right\rangle \times B e_{2 n}\left(\xi, q_{2 n, m}\right) c e_{2 n}\left(\eta, q_{2 n, m}\right) / \beta_{p}^{2} C_{2 n, m} \\
& N_{\theta}=0
\end{aligned}
$$

Using Eqs (5.6) in Eq.(4.2), we obtain the expression for $\omega$ as follows

$$
\begin{aligned}
& \omega=\frac{2 \alpha E}{D \ell(1-v)} \sum_{n=0}^{\infty} \sum_{m=1}^{\infty} \sum_{p=1}^{\infty}\left[(-1)^{p}-1\right]\left\langle\int_{0}^{t} E_{\alpha}\left\{\left[-\kappa\left(\lambda_{2 n, m}^{2}+\beta_{p}^{2}\right)\right] u^{\alpha}\right\} \times\right. \\
& \left.\times\left[-\frac{4 \kappa}{c^{2}} \pi A_{0}^{2 n} B e_{2 n}\left(\xi_{0}\right) \hat{\bar{f}}\left(q_{2 n, m}, \beta_{p}, t-u\right)\right] d u\right\rangle \times B e_{2 n}\left(\xi, q_{2 n, m}\right) c e_{2 n}\left(\eta, q_{2 n, m}\right) / \lambda_{2 n, m}^{2} \beta_{p}^{2} C_{2 n, m}
\end{aligned}
$$

Using Eqs (5.6) and (5.7) in Eq.(4.4), the resultant bending moments per unit width $\left(M_{i j}\right)$ are as follows 


$$
\begin{aligned}
& M_{\xi}=-\frac{2 \alpha E}{\ell(1-\mathrm{v})} \sum_{n=0}^{\infty} \sum_{m=1}^{\infty} \sum_{p=1}^{\infty}\left[(-1)^{p}-1\right]\left\langle\int_{0}^{t} E_{\alpha}\left\{\left[-\kappa\left(\lambda_{2 n, m}^{2}+\beta_{p}^{2}\right)\right] u^{\alpha}\right\} \times\right. \\
& \left.\left.\times-\frac{4 \kappa}{c^{2}} \pi A_{0}^{2 n} B e_{2 n}\left(\xi_{0}\right) \hat{\bar{f}}\left(q_{2 n, m}, \beta_{p}, t-u\right)\right] d u\right\rangle \times\left\langleh ^ { 2 } \left\{\left[ B e^{\prime \prime}{ }_{2 n}\left(\xi, q_{2 n, m}\right) c e_{2 n}\left(\eta, q_{2 n, m}\right)+B e_{2 n}\left(\xi, q_{2 n, m}\right) \times\right.\right.\right. \\
& \left.\times c e_{2 n}\left(\eta, q_{2 n, m}\right)\right]-\frac{c^{2} h^{2}(1-v)}{2}\left[\sinh 2 \xi B e_{2 n}^{\prime}\left(\xi, q_{2 n, m}\right) \times c e_{2 n}\left(\eta, q_{2 n, m}\right)+\right. \\
& \left.\left.\left.+\sin 2 \eta B e_{2 n}\left(\xi, q_{2 n, m}\right) c e^{\prime}{ }_{2 n}\left(\eta, q_{2 n, m}\right)\right]\right\}-\lambda_{2 n, m}^{2} B e_{2 n}\left(\xi, q_{2 n, m}\right) c e_{2 n}\left(\eta, q_{2 n, m}\right)\right\rangle / \lambda_{2 n, m}^{2} \beta_{p}^{2} C_{2 n, m}, \\
& M_{\eta}=-\frac{2 \alpha E}{\ell(1-v)} \sum_{n=0}^{\infty} \sum_{m=1}^{\infty} \sum_{p=1}^{\infty}\left[(-1)^{p}-1\right]\left\langle\int_{0}^{t} E_{\alpha}\left\{\left[-\kappa\left(\lambda_{2 n, m}^{2}+\beta_{p}^{2}\right)\right] u^{\alpha}\right\} \times\right. \\
& \left.\left.\times-\frac{4 \kappa}{c^{2}} \pi A_{0}^{2 n} B e_{2 n}\left(\xi_{0}\right) \hat{\hat{f}}\left(q_{2 n, m}, \beta_{p}, t-u\right)\right] d u\right\rangle \times\left\langleh ^ { 2 } \left\{\left[ v B e^{\prime \prime}{ }_{2 n}\left(\xi, q_{2 n, m}\right) c e_{2 n}\left(\eta, q_{2 n, m}\right)+B e_{2 n}\left(\xi, q_{2 n, m}\right) \times\right.\right.\right. \\
& \left.\times c e_{2 n}\left(\eta, q_{2 n, m}\right)\right]+\frac{c^{2} h^{2}(1-v)}{2}\left[\sinh 2 \xi B e_{2 n}^{\prime}\left(\xi, q_{2 n, m}\right) \times c e_{2 n}\left(\eta, q_{2 n, m}\right)+\right. \\
& \left.\left.\left.-\sin 2 \eta B e_{2 n}\left(\xi, q_{2 n, m}\right) c e^{\prime}{ }_{2 n}\left(\eta, q_{2 n, m}\right)\right]\right\}-\lambda_{2 n, m}^{2} B e_{2 n}\left(\xi, q_{2 n, m}\right) c e_{2 n}\left(\eta, q_{2 n, m}\right)\right\rangle / \lambda_{2 n, m}^{2} \beta_{p}^{2} C_{2 n, m}, \\
& M_{\xi \eta}=-\frac{2 h^{2} \alpha E}{\ell} \sum_{n=0}^{\infty} \sum_{m=1}^{\infty} \sum_{p=1}^{\infty}\left[(-1)^{p}-1\right]\left\langle\int_{0}^{t} E_{\alpha}\left\{\left[-\kappa\left(\lambda_{2 n, m}^{2}+\beta_{p}^{2}\right)\right] u^{\alpha}\right\} \times\right. \\
& \left.\left.\times-\frac{4 \kappa}{c^{2}} \pi A_{0}^{2 n} B e_{2 n}\left(\xi_{0}\right) \hat{\bar{f}}\left(q_{2 n, m}, \beta_{p}, t-u\right)\right] d u\right\rangle \times \\
& \times\left\langle\sin 2 \eta B e_{2 n}\left(\xi, q_{2 n, m}\right) c e_{2 n}\left(\eta, q_{2 n, m}\right)+\sinh 2 \xi B e_{2 n}^{\prime}\left(\xi, q_{2 n, m}\right) \times\right. \\
& \left.\times c e^{\prime}{ }_{2 n}\left(\eta, q_{2 n, m}\right)-\frac{2}{c^{2} h^{2}} B e_{2 n}^{\prime}\left(\xi, q_{2 n, m}\right) c e_{2 n}^{\prime}\left(\eta, q_{2 n, m}\right)\right\rangle / \lambda_{2 n, m}^{2} \beta_{p}^{2} C_{2 n, m} .
\end{aligned}
$$

MATHEMATICA software was used for the graphic analysis of the thermal stresses which are calculated easily by using Eqs (5.6) to Eq.(5.10) in Eq.(4.7).

\section{Results and discussion}

Dimensions. For the sake of convenience, we choose the physical parameter as [23] $\bar{\xi}_{i}=1.5 \mathrm{~m}, \bar{\xi}_{o}=0.75 \mathrm{~m}$, $\bar{\ell}=0.05 \mathrm{~m}, c=e=0.5 \mathrm{~m}$ and reference temperature $T_{0}$ as $150^{\circ} \mathrm{C}$. The $q_{2 n, m}=1.331,1.572,2.628,4.242$, $6.235,6.621,8.728,9.642,10.564,12.455,13.846,15.235,17.731,19.452,20.323,23.235,24.553,24.458$, $25.231,27.568$ are the positive and real roots of the $B e_{2 n}\left(\xi_{0}, q_{2 n, m}\right)=0$.

\section{Numerical results, discussion and remarks}

The numerical calculation has been carried out for a copper (pure) elliptical disk with the following material properties: thermal diffusivity $\kappa=112.34 \times 10^{-6} \mathrm{~m}^{2} \mathrm{~s}^{-1}$, thermal conductivity $\lambda=386(\mathrm{~W} / \mathrm{mK})$, density 
$\rho=2954 \mathrm{~kg} / \mathrm{m}^{3}$, Poisson's ratio $\mathrm{v}=0.35$, thermal expansion coefficient $\alpha=16.5 \times 10^{-6} \rho \mathrm{C}$ and modulus of elasticity $E=70 \mathrm{GPa}$.

The graphs are plotted for the fractional-order parameter $\alpha=0.5 ; 1 ; 1.5$ and 2 depicting different conductivity and fixed time $t=0.5$. Figures 2-6 depict the distributions of temperature, radial stress and angular stress along the radial direction for various values of the fractional-order parameter $\alpha$. The numerical calculation has been carried out in MATHEMATICA programing.

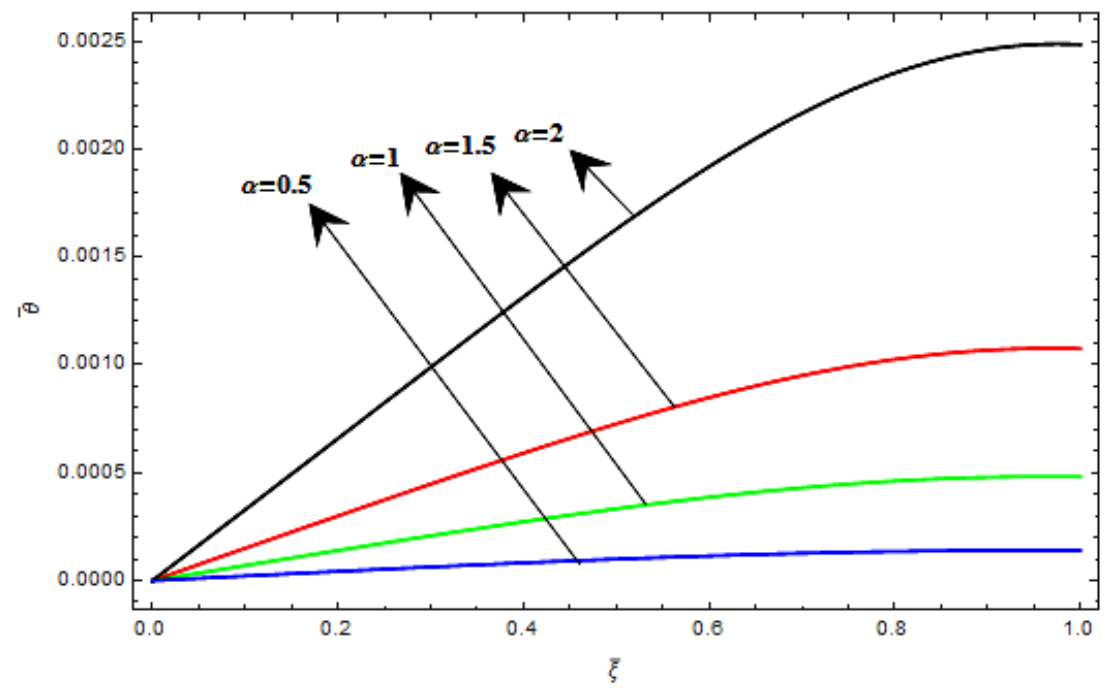

Fig.2. Temperature distribution function.

Figure 2 represents the temperature distributions along the $\bar{\xi}$-direction for different $\alpha$. For the cases $\alpha=0.5 ; 1 ; 1.5$ and 2 , the values of the temperature show an increase with respect to $\bar{\xi}$ and it attains the highest value according to the value of $\alpha$. It is clear that increasing the values of the fractional order parameter causes increasing the value of the temperature. Due to the sectional heat supply at $\bar{\xi}=1$ of the plate, the effect of temperature at the outer edge is observed.

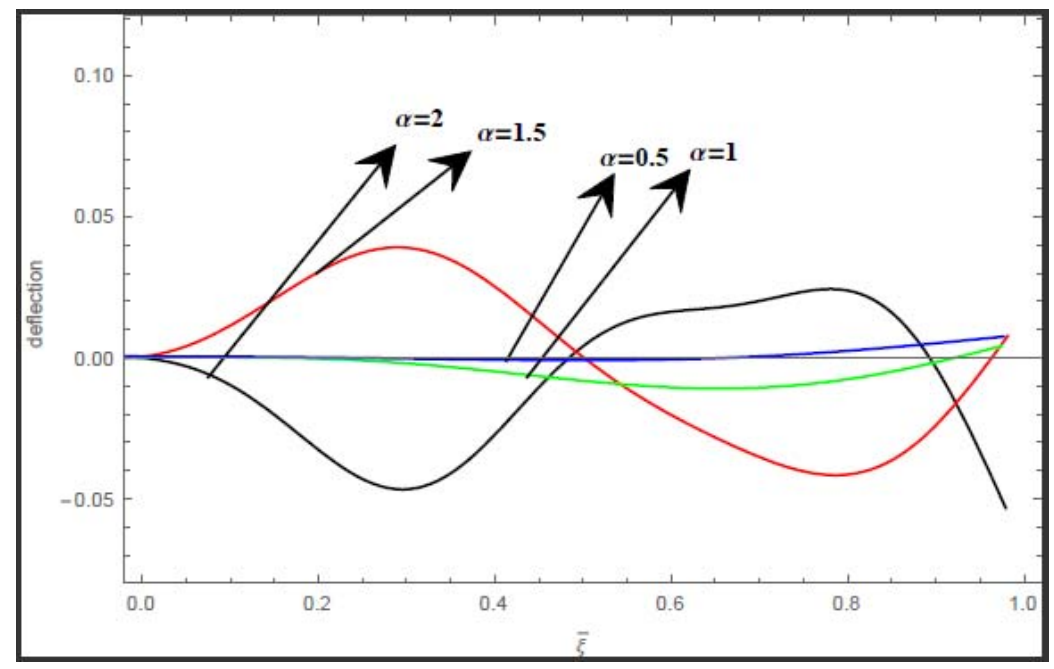

Fig.3. Thermal deflection along $\bar{\xi}$ - direction for different $\alpha$. 
The thermal deflection along the $\bar{\xi}$ - direction for different $\alpha$ is sinusoidal in nature. The figure shows that thermal deflection is zero at the inner boundary which satisfies the boundary condition $\bar{\omega}=0$, at $\bar{\xi}=\bar{\xi}_{i}$, then decreases gradually along the radial direction for various values of $\alpha$

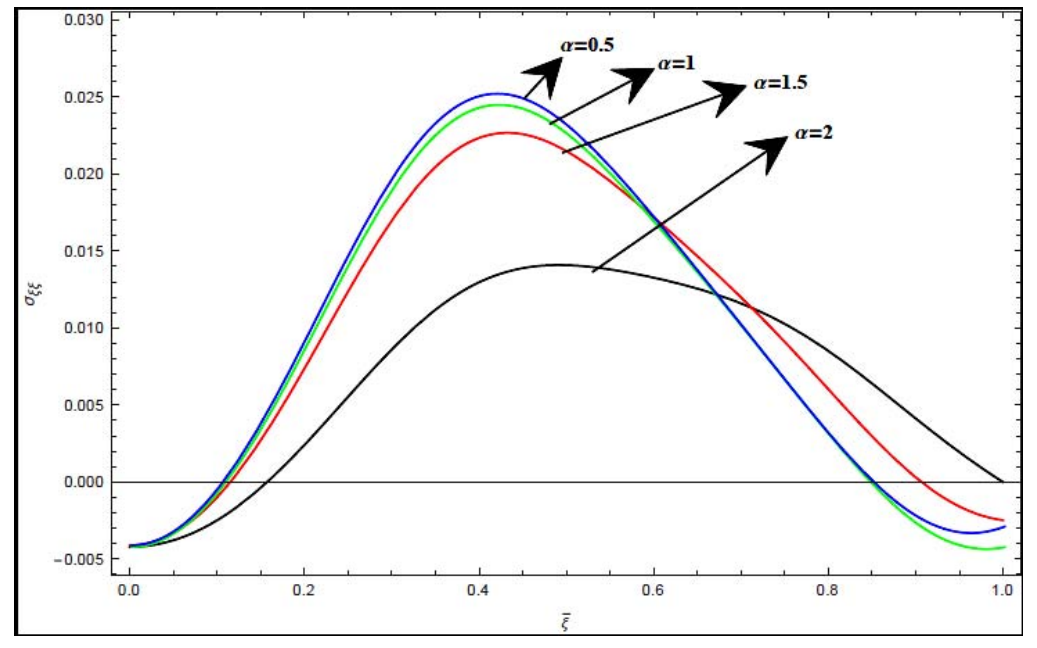

Fig.4. Radial stresses function along $\bar{\xi}$ - direction for the different $\alpha$.

Figure 4 represents the radial stress distributions along along the $\bar{\xi}$-direction for different $\alpha$. The nature of the graph is sinusoidal and is highest at the middle core of the disc and represents the maximum amplitude of the wave propagation for the different values of $\alpha$.

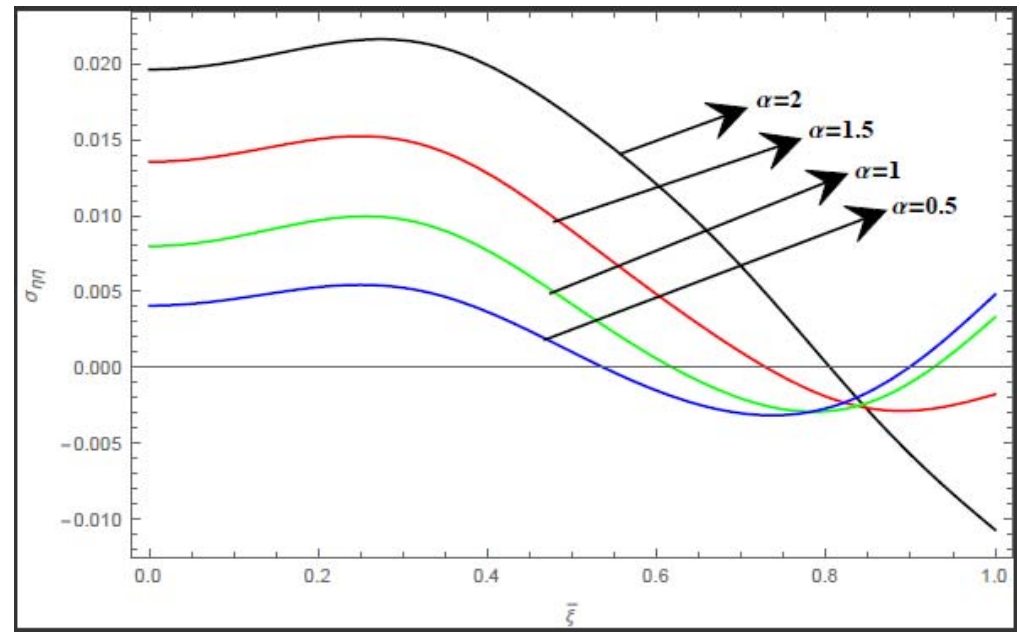

Fig.5. Tangential stresses along $\bar{\xi}$. - direction for different $\alpha$.

Figure 5 illustrates tangential stresses along the $\bar{\xi}$. - direction for different $\alpha$. It can be seen that the stress increases gradually with the increase in the radius and attains certain maxima and starts decreasing in outward direction. 


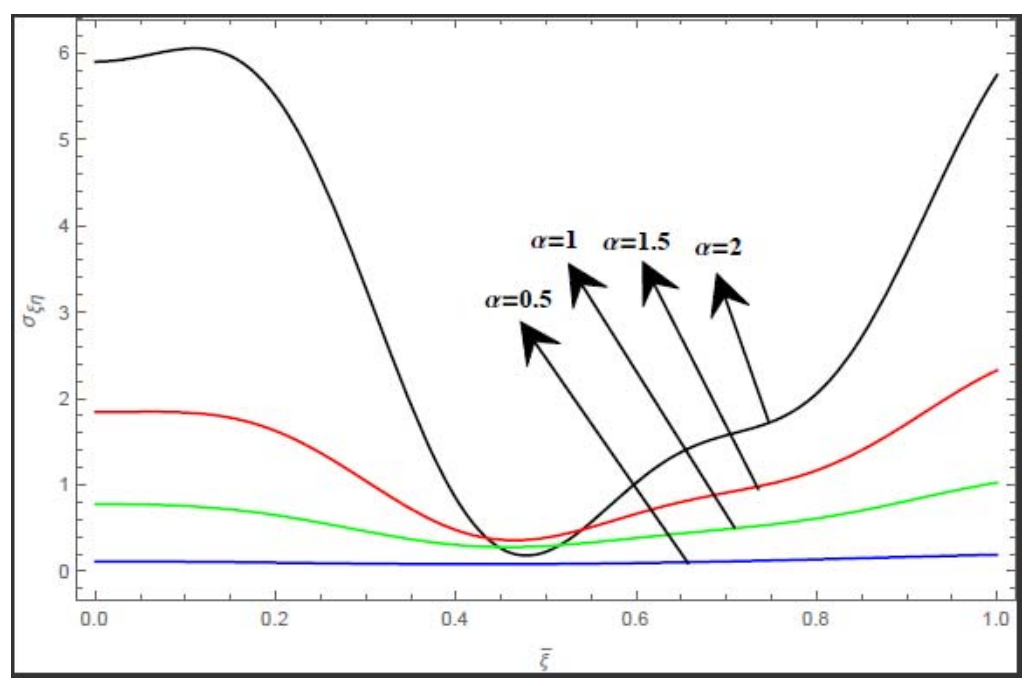

Fig.6. Shear stresses along $\bar{\xi}$. - direction for different $\alpha$.

Figure 6 show shear stresses along the $\bar{\xi}$. direction for different $\alpha$. It can be seen that the stress decreases gradually with the increase in the radius and attains certain minima and starts increasing in outward direction.

\section{Conclusion}

This article is based on the uncoupled theoretical treatment of the time fractional-order heat conduction equation by neglecting the inertia term in the equation of motion. The integral transform methods are used to determine temperature distribution and the deflection in the form of ordinary and modified Mathieu functions. Also, the effect on bending stresses was investigated in the context fractional heat. It is observed that with an increase in the value of the fractional parameter corresponding speed of the waves increases, which shows that the fractional order parameter and time have a significant effect on the temperature distribution and the thermal deflection. Thus the analytical technique proposed here predicts a retarded response to physical stimuli and is widely applicable.

\section{Nomenclature}

$$
\begin{aligned}
2 a_{i} & - \text { inner diameter of semi-major axis } \\
2 a_{o} & - \text { outer diameter of semi-major axis } \\
2 b_{i} & - \text { inner diameter of semi-minor axis } \\
2 b_{o} & - \text { outer diameter of semi-minor axis } \\
C & - \text { calorific capacity } \\
C e_{2 n}(\xi, q) & - \text { modified Mathieu function of first kind of order } n \\
c e_{2 n}(\eta, q) & - \text { ordinary Mathieu function of first kind of order } n \\
2 c & - \text { denotes the distance between their common foci } \\
D & - \text { flexural stiffness of the disk } \\
E & - \text { Young's modulus } \\
E_{\alpha}(z) & - \text { Mittag-Leffler function } \\
M_{i j} & - \text { resultant bending moments per unit width } \\
M_{\theta}(\xi, \eta, t) & - \text { thermally induced resultant force } \\
N_{i j} & - \text { resultant forces }
\end{aligned}
$$




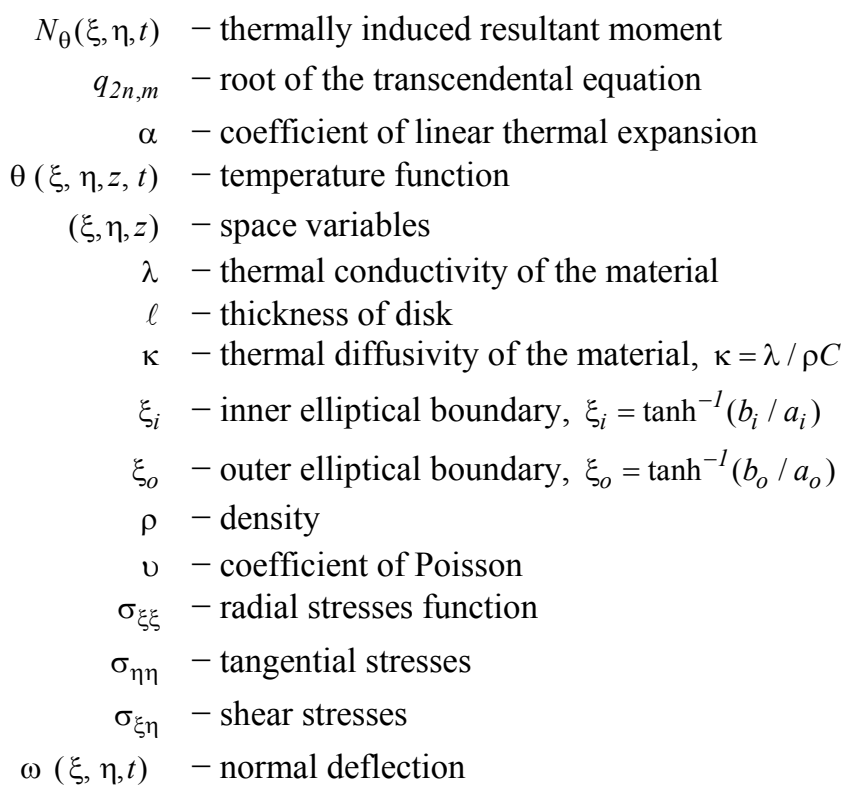

\section{References}

[1] Abel N.H. (1823): Solution de Quelques Problèmes à I'aide D'intègrales Dèfinites. - Werkee, vol.1, pp.10.

[2] Caputo M. and Mainardi F. (1971): A new dissipation model based on memory mechanism. - Pure Appl. Geophys., vol.91, pp.134-147.

[3] Caputo M. and Mainardi F. (1971): Linear models of dissipation in an elastic solids. - Rivis ta del Nuovo ciment, vol.1, pp.161-198.

[4] Caputo M. (1974): Vibration on an infinite viscoelastic layer with a dissipative memory. - J. Acous. Soc. Am., vol.56, pp.897-904.

[5] Ning T.H. and Jiang X.Y. (2011): Analytical solution for the time-fractional heat conduction equation in spherical coordinate system by the method of variable separation. - Acta Mechanica Sinica, vol.27, No.6, pp.994-1000.

[6] Lenzi E.K., Mendes R.S., Gonçalves G., Lenzi M.K. and da Silva L.R. (2006): Fractional diffusion equation and Green function approach: Exact solutions. - Physica A 360, pp.215-226.

[7] Povstenko Y. (2013): Fractional heat conduction in an infinite medium with a spherical inclusion. - Entropy, vol.15, pp.4122-4133.

[8] Povstenko Y. (2015): Fractional Thermoelasticity. - Switzerland: Springer.

[9] Povstenko Y. (2016): Fractional heat conduction in a space with a source varying harmonically in time and associated thermal stresses. - J. Therm. Stresses, vol.39, No.11, pp.1442-1450.

[10] Abbas I.A. (2015): Eigen value approach on fractional order theory of thermoelastic diffusion problem for an infinite elastic medium with a spherical cavity. - Applied Mathematical Modelling, vol.39, pp.6196-6206 (2015)

[11] Warbhe S.D., Tripathi J.J., Deshmukh K.C. and Verma J. (2017): Fractional heat conduction in a thin circular plate with constant temperature distribution and associated thermal stresses. - Journal of Heat Transfer, vol.139 / 044502-1-4.

[12] Youssef H.M. (2010): Theory of fractional order generalized thermoelasticity. - J. Heat Transfer, vol.132, pp.17 .

[13] Youssef H.M. (2012): Two-dimensional thermal shock problem of fractional order generalized thermoelasticity. - Acta Mech., vol.223, pp.219-1231. 
[14] Khobragade N.L. and Lamba N.K. (2019): Modeling of thermoelastic hollow cylinder by the application of fractional order theory. - Research and Reviews: Journal of Physics; vol.8, Nio.1, pp.46-57.

[15] Tripathi J.J., Kedar G.D. and Deshmukh K.C. (2016): Dynamic problem of fractional order thermoelasticity for a thick circular plate with finite wave speeds. - J. Therm. Stresses, vol.39, No.2, pp.220-230.

[16] Kukla S. and Siedlecka U. (2017): An analytical solution to the problem of time-fractional heat conduction in a composite sphere. - Bulletin of the Polish Academy of Sciences Technical Sciences, vol.65, No.2, pp.179-186.

[17] Khobragade N.L. and Lamba N.K. (2019): Thermal deflection and stresses of a circular disk due to partially distributed heat supply by application of fractional order theory. - Journal of Computer and Mathematical Sciences, vol.10, No.3, pp.429-437.

[18] Khobragade N.L. and Lamba N.K. (2019): Study of thermoelastic deformation of a solid circular cylinder by application of fractional order theory. - Journal of Computer and Mathematical Sciences, vol.10, No.3, pp.438-444.

[19] McLachlan N.W. (1945): Heat conduction in elliptical cylinder and an analogous electromagnetic problem. Philosophical Magazine, vol.36, pp.600-609.

[20] McLachlan N.W. (1947): Theory and Application of Mathieu function. - Oxford: Clarendon Press.

[21] Dhakate T., Vinod Varghese and Lalsingh Khalsa (2017): Integral transform approach for solving dynamic thermal vibrations in the elliptical disk. - Journal of Thermal Stresses, DOI: 10.1080/01495739.2017.1285215.

[22] Gupta R.K. (1964): A finite transform involving Mathieu functions and its application. - Proc. Net. Inst. Sc., India, Part A, vol.30, pp.779-795.

[23] Bhad P., Vinod Varghese and Lalsingh Khalsa (2017): Heat source problem of thermoelasticity in an elliptic plate with thermal bending moments. - Journal of Thermal Stresses, vol.40, No.1, pp.96-107.

[24] Dhakate T., Vinod Varghese and Lalsingh Khalsa (2018): A simplified approach for the thermoelastic large deflection in the thin clamped annular sector plate. - Vol. 41, No.2, pp.271-285.

[25] Ishteva M., Scherer R. and Boyadjiev L. (2005): On the Caputo operator of fractional calculus and C-Laguerre functions. - Mathematical Sciences Research Journal, vol.9, No.6, pp.161-170.

[26] Haubold H.J., Mathai A.M. and Saxena R.K. (2011): Mittag-Leffler functions and their applications. - Journal of Applied Mathematics, paper ID 298628.

[27] Bhad P.P. (2017): Study of thermoelastic problems on elliptical objects with an internal heat source. - PhD Dissertation - Gondwana University, Gadchiroli, India.

Received: July 13, 2019

Revised: March 20, 2020 